\title{
Is My University Inclusive? Towards a Multi-Domain Instrument for Sustainable Environments in Higher Education
}

\author{
Giulia BENCINI $^{\mathrm{a}, 1}$, Alberto ARENGHI ${ }^{\mathrm{b}}$, Ilaria GAROFOLO ${ }^{\mathrm{c}}$ \\ ${ }^{\text {a }} \mathrm{Ca}$ ' Foscari University of Venice \\ ${ }^{\mathrm{b}}$ University of Brescia \\ ${ }^{\mathrm{c}}$ University of Trieste
}

\begin{abstract}
We present a pilot study on three Italian Universities using a multidomain set of indicators for Inclusion. The indicators are expressed in the coding system of the International Classification of Functioning Disability and Health ICF - (World Health Organization, 2001).

We selected three medium-sized Italian Universities: Brescia, Trieste and Venice. We combined a student-centered Universal Design philosophy for the built environment and Universal Design for Learning for the instructional environment. We identified four ICF Environmental Chapters (E1, E3, E4 and E5) and made them specific to the Academic context. Within the four Environmental Chapters targeting the physical, instructional, cultural, communicative, social and recreational domains we developed a 35 -item checklist to fill out. The indicators were qualitative, quantitative or a mixture of the two. The three Universities shared the same instruments. Our main finding is that, although accommodations for students with disabilities exist as mandated by Italian law, the prevailing implementation is an individual accommodation based approach, rather than a universal design approach for the benefit to the greatest extent of the student population.
\end{abstract}

Keywords. Inclusion; ICF; Higher Education; Sustainable Development Goals

\section{Introduction}

Inclusion of a diverse student population has increasingly been embraced by Higher Education Institutions worldwide. The United Nations 2030 Agenda for Sustainable Development for the first time explicitly mentions inclusion of children and adults with disabilities among its goals both for education (Sustainable Development Goal 4: Access to quality education for all). Access and inclusion in higher education require changes to the environments in which higher education takes place. We believe that Inclusive Higher Education benefits from person-centered approaches that recognize human diversity from the get-go. People differ along many possible dimensions: physical, linguistic, cognitive, cultural. At the same time Universities must also uphold higher education standards that may differ from mandatory secondary school outcomes. Finally, because many of the environments in which European Universities are located were not designed to be accessible and in many cases Universities are located in historical

\footnotetext{
${ }^{1}$ Giulia Bencini, Department of Linguistics and Comparative Cultural Studies, Ca Foscari University Venice, Ca Bembo, Fondamenta Toffetti, Dorsoduro, Venice, Italy; E-mail: giulia.bencini@unive.it.
} 
buildings and areas protected by historical conservation laws, there is a need in many cases to re-design learning environments (spaces and services) in higher education.

In this paper we present the results of a pilot study on three mid-sized public Italian Universities aimed at developing a set of multi-domain indicators to measure Inclusion and Universal Design [1]. The indicators are expressed adopting the conceptual framework and coding system offered by the International Classification of Functioning Disability and Health - ICF - [2] combined with a Universal Design student-centered philosophy for the built environment and Universal Design for Learning for the instructional environment. The multi-domain instrument aims to provide a valid and measurable set of indicators for inclusion in Higher Education to be shared at the micro (e.g., departmental), meso (University-wide) and macro (national) level. The instrument is intended to inform and assist governance bodies, academic staff, building managers, curriculum developers, and the entire community of stakeholders in Higher Education better implement mainstreaming inclusive policies.

The pilot study used a survey-based methodology with a set of questions aimed at obtaining information about learning facilities, classrooms, online platforms, signage and navigation, teaching methods and materials, language and communication, systems-wide policies and attitudes towards diversity and inclusion, cultural, recreational and public engagement activities.

\subsection{Why the ICF?}

The reason we chose the ICF framework lies in its neutrality with respect to disability. Although the ICF has historically been adopted in clinical settings, we believe that its use can be extended to educational settings, including Higher Education. The ICF applies to all human beings and offers a fine-grained ontology of the kinds of things human beings do in their physical and social environments. Of course, the ICF is grounded in the body. As a person-centered classification system the ICF includes both a description of a person's body structures and functions (e.g., motor and sensory functions, special mental functions such as language) the activities that a person typically engages in such as, for example, communicating via spoken, written or visual media, the domains of life participation such as education, culture, sports, civic life. As a neutral coding system it lets individual professionals exercise their domain of expertise on the one hand, while at the same time allowing for communication across disciplines when the interest is in a human-centered approaches to design. The ICF is useful in Higher Education because it allows governance bodies and planners of the built and the learning environment consider the design impact of differing characteristics of students from various backgrounds and ages; students navigating the built environment and the virtual environment without sight or hearing, or even with neither sight nor hearing; varying language backgrounds and competencies in accessing and understanding information presented in print media, audio, video, communication and websites; the consequences of disease or trauma; the challenges for students with limited mobility or agility as they seek to participate in academic life (lectures, events, international mobility and study abroad).

Our instrument is novel in several ways: first, we adopt a broader definition of "Environment" than commonly understood: within a human-centered design philosophy we take environment to encompass not just the physical, but also the instructional, communicative, cultural and social aspects of the environment, including people's attitudes. Second, following the terminology adopted by the ICF we take disability to be 
a neutral term to refer to any limitations in either functioning, activities or participation [2] resulting from the interaction between personal characteristics or circumstances and the environment. Third, we make an effort propose a framework suitable for crossdisciplinary collaboration and adopt a neutral language to be shared by all actors who are committed to sustainability and inclusion in Higher Education. Fourth, we aim for the indicators to be measurable so as to guide University governance bodies at the local, regional and national level assess, implement inclusive policies and monitor progress. Finally, we aimed for the instrument to be easy to fill out, with minimal burden on the administrative staff.

\section{Method}

\subsection{Participating Institutions}

For the pilot phase we selected three medium-sized Italian public Universities: the University of Brescia, the University of Trieste and Ca Foscari University of Venice. The three Universities have undergraduate and graduate programs that span across the liberal-arts and humanities, the physical, biological and social sciences, medicine, architecture and engineering. All three universities have many buildings in historical sites and are located in historical contexts with accessibility challenges (e.g., Venice). Although the number of participating institutions is limited, they were selected so as to include different representative scenarios and challenges with respect to the topographical, physical, technological and cultural environment. The participating Universities comply with Italian National Legislation with respect to services and provisions for students with disabilities and specific learning impairments. Italian Law mandates that each public University appoints a Rector's Delegate for Disabilities and Specific Learning Impairments. This individual is a full-time faculty member who acts as a policy advisor and overseer of all provisions that concern students with disabilities. In each one of the three participating institutions the authors either currently or formerly held the position of Rector's Delegate. Italian Delegates for Disabilities are also members of a National Committee of University Delegates (CNUDD) with regular meetings and frequent exchanges among Delegates and student disability support offices. The set of indicators for Inclusion in [1] was presented to the CNUDD Assembly and governing board and received positive feedback. The assembly agreed to start pilot testing the instrument on three universities with the idea that more Universities would participate in the future.

\subsection{Definition and selection of the indicators}

The set of indicators was converged upon by first agreeing on a set of learning environments typical of Italian Universities, from a student-centered perspective. The set of environments is intentionally circumscribed to primary teaching and learning environments on University premises and does not include Housing. This choice is motivated by the national context where student housing and accommodation is typically outside of the University's jurisdiction.

We selected the following macro-domains (environments) and identified the corresponding Chapter within the ICF set of Environments: 1) the built environment; 
2) ICT and the web; 3) the learning environment broadly construed to cover teaching and learning, also including educational staff (professors and support staff), learning technology and technology for education; 4) language and communication; 5) systems, policies and attitudes. Within the macro-environments we selected sets of qualitative and quantitative indicators to be collected and assessed.

\subsection{Instruments}

Across academic institutions the seven ICF domains typically fall under different delegates and or institutional bodies. In order to facilitate data collection and replicability across institutions we created a 35 items questionnaire with specific questions for each macro-domain. Example questions for three environmental domains the built environment, the learning environment, and the communicative environment is provided in Table 1. The full set of questions is available upon request.

Table 1. Example questions concerning the built, learning and communicative environments

\begin{tabular}{l} 
Environment Queried \\
\hline Built Environment (E 150) \\
What percentage classrooms, labs, libraries and facilities meet accessibility standards? \\
What percentage classrooms, labs, libraries have adaptable seating positions? \\
What percentage signage and way-finding are universally designed? \\
What percentage doors, elevators, etc. are universally designed? \\
Learning Environment: Technology, Courses, and Instructional Professionals (E130, E 360) \\
What percentage of classrooms is equipped with audio-video recording and subtitling software? \\
In what percentage of courses learning objectives and assessment designed following UD principles? \\
Is there training on the use of technology for universal design for educational and technical staff? \\
Communicative Environment: communication for learning, communicating via the web, document \\
policies, events and public engagement \\
Is communication addressed to students accessible with software and designed to be comprehensible? \\
Is the website accessible and designed according to UD principles?
\end{tabular}

\subsection{Procedure}

In each participating University the authors distributed the questionnaires to each office or Delegate responsible for the corresponding domain. Participating offices were informed of the scientific aims of the study, namely to assist in the construction of an easy to use questionnaire to measure the extent to which our environments were universally designed for inclusion. The questionnaire was preceded by a paragraph providing the definition of Universal Design contained in the United Nations Convention of the Rights of Persons with Disabilities [3]. Crucially, the questions were formulated in such a way as to not require any prior knowledge of Universal Design. For example, a question like "What percentage of events organized by the University (e.g. Beginning of the Academic year, Graduation and Commencement Ceremonies) have accessibility features such as Subtitling, sign language interpreters?" The questionnaire covers diversified environmental domains pertaining to different offices, which may collect quality assurance information in different forms. We believe that one of the desiderata of an instrument to measure inclusion is ease of completion including time to complete. For this reason, we decided that a reasonable time for completion was one week. The Rector's Delegate for Disabilities and Specific Learning Impairments in each University was responsible for collecting the data and filling out questions that could be filled out 
without additional involvement of the administrative staff. This is an important feature of our instrument, as University offices are already subject to many quality assurance protocols and gathering more data for the purposes of getting a multidimension wholistic view of Inclusion should not be an excessive burden to administration.

The survey aimed at gathering information pertaining to the five environments directly relevant to students' university experience according to the macro-domains listed above. Because of existing Italian regulations for the built environment and for the web we predicted that the data for the corresponding domains (built environment, ICT and the web) would be easier to obtain and that more progress towards Accessibility should be visible in these domains, but not necessarily in a Universal Design perspective.

\section{Results and Discussion}

The survey data for the three participating Universities are presented in Table 2. All three participating universities showed numerically high percentages for accessibility to classrooms, lecture halls, libraries and labs built environment. Much lower scores were reported for less tangible aspects such as navigation facilitators and universally designed signage, universally designed door opening and elevators. Noticeably low was also the percentage of adaptable seating in classrooms and labs (0-25\% in all three institutions).

Institutions also reported a high percentage of ICT equipment with accessibility features, but relatively low percentages for more advanced software and technology. For the learning environment which included technology in classrooms (captioning, audio, video, etc.) and online learning platforms, curricula, learning objectives, learning materials data in all three of our universities was not readily available. This finding suggests that, although accommodations for students with disabilities exist as mandated by Italian law, the prevailing implementation is an individual accommodation based approach, rather than a Universal Design approach for the benefit to the greatest extent of the student population.

Table 2. Survey results for the participating universities by environmental domain.

\begin{tabular}{|c|c|c|c|c|}
\hline Questionnaire & Environmental Indicator & U1 & U2 & $\mathbf{U 3}$ \\
\hline & ICT Environment, web (E 125, E130) & & & \\
\hline 1 & Basic accessibility software on computers & $100 \%$ & $100 \%$ & $100 \%$ \\
\hline $2-3$ & Dedicated hardware and software & $0-25 \%$ & $0-25 \%$ & $0-25 \%$ \\
\hline \multirow[t]{2}{*}{34} & Accessible and UD designed Web Environment & yes & no & no \\
\hline & $\begin{array}{l}\text { Learning Environment: Technology, Courses, } \\
\text { and Instructional Professionals (E130, E 360) }\end{array}$ & & & \\
\hline 6 & $\begin{array}{l}\text { Classrooms Equipped with recording and } \\
\text { subtitling software }\end{array}$ & $0-25 \%$ & $0-25 \%$ & $0-25 \%$ \\
\hline 7 & $\begin{array}{l}\text { Courses designed multimodally (audio, video, } \\
\text { transcription) }\end{array}$ & NA & NA & $0-25 \%$ \\
\hline 10 & $\begin{array}{l}\text { Learning objectives, assessment designed } \\
\text { following UD principles }\end{array}$ & NA & NA & $0-25 \%$ \\
\hline \multirow[t]{2}{*}{$8-9-24-25$} & Training for educational and technical staff & no & no & no \\
\hline & Built Environment (E 150) Safety (E 545) & & & \\
\hline 14 & Accessible classrooms locations and spaces & $76-100 \%$ & $76-100 \%$ & $26-50 \%$ \\
\hline 20 & Adaptable seating in classrooms, labs, libraries & $0-25 \%$ & $26-50 \%$ & $0-25 \%$ \\
\hline $15-16-17-18-19$ & UD signage and wayfinding & $0-25 \%$ & $0-25 \%$ & $0-25 \%$ \\
\hline $21-22$ & UD designed doors, elevators, etc. & $0-25 \%$ & $0-25 \%$ & $0-25 \%$ \\
\hline 29 & Multimodal Hazard and Safety Information & $51-75 \%$ & $76-100 \%$ & $26-50 \%$ \\
\hline
\end{tabular}




\begin{tabular}{|c|c|c|c|c|}
\hline \multirow{3}{*}{32} & \multicolumn{4}{|l|}{ Systems and Policies (E5) } \\
\hline & Inclusion stated in University Strategic Plan & no & yes & yes \\
\hline & $\begin{array}{l}\text { Public Engagement, Communication and } \\
\text { Events for the Community at large }\end{array}$ & & & \\
\hline $26-27$ & Events with interpreters, subtitling & $0-25 \%$ & $0-25 \%$ & $0-25 \%$ \\
\hline
\end{tabular}

Our predictions that universities would be doing better for domains where accessibility standards exist and are mandated by law, were confirmed.

The results of our pilot study demonstrated the effectiveness of our multi-domain instrument in providing an overarching picture of how well universities are doing when addressing the complex nature of inclusion policies and mainstreaming of students disabilities. The main finding is that whereas data are more readily obtainable for domains were regulations exist (accessibility to the built environment, ICT accessibility and the web) none of the universities had readily available data concerning courses, assessment and materials for learning. None of the universities had, prior to Spring 2020, regular training for their teaching staff on accessibility, technology for inclusion, universal design. As discussed in Section 4, The Covid-19 Emergency put universities worldwide to an unprecedented "stress test".

One challenge revealed during the pilot phase was the difficulty obtaining data from different university organizational units (built environment, ICT, teaching and learning) and the absence of data on accessibility and inclusion when it comes to teaching and learning. We should point out, however, that individual provisions for students with disabilities exist in all three universities. Our point is that the provisions are based on an individual needs approach, not a Universal Design approach. Our work brought to light the usefulness of establishing university-wide boards focused on diversity, equity and inclusion to engage all actors with responsibilities for specific domains. One of the three participating universities had such a board already in place. The board, coordinated by the Rector's Delegate for Disabilities, provided a receptive forum to introduce the Universal Design philosophy in different domains. The board also currently serves as an advisory board to the director to plan future environmental interventions aimed at mainstreaming and inclusion, in line with sustainable development goals. The connection between inclusion and sustainability is also being reflected at the national level. Recently a new working group on Inclusion and Social Justice was formed within the Italian Network for Sustainable Universities.

The implementation phase of our initial instrument brought to light one limitation of using the ICF environmental chapters as currently coded. Although the ICF provides a fine-grained classification system for person-centered functions and activities, it appears to be as of yet too coarse to uniquely specify environmental characteristics of higher education. For example, in order to identify "professors" and "teaching staff" we had to resort to the very general E360 chapter ("supports and relationships: other professionals").

\section{Universities shift to remote learning in response to the Covid-19 Epidemic and (some) desirable consequences for Universal Design}

Since administering our Questionnaire, an unprecedented shift has taken place in the teaching, learning and assessment practices in Higher Education as a consequence of the Covid-19 Epidemic starting in Spring 2020. Higher Education was put to an 
unprecedented stress test, as all courses and examinations were suddenly shifted to distance learning. This in turn has led to widespread investments in all three participating institutions in ICT infrastructure for remote or dual learning, and training of faculty, staff and students on the use of different platforms.

At the end of the 2020 spring semester, we conducted a survey with students with disabilities and specific learning disorders to assess whether any difficulties or accessibility issues emerged during the semester, and to assess their degree of satisfaction with remote learning during the lockdown phase [4]. The survey revealed a general preference for in-class learning as the best means to participate in university life and to have opportunities for social interaction with instructors and peers. Students' socio-emotional difficulties emerged, connected to decreased social interaction with faculty and with peers. At the same time, students' responses also revealed positive responses to the increased use of technology. Many desirable features connected to the "built in" potential of ICT to implement Universal Design for Learning became evident. Students expressed an appreciation for the diffuse use of video-recordings of university lectures and the greater availability of digitalized and multimodal material. They also appreciated the use of subtitling and captioning, and the availability of remote signed language interpretation services. The fact that everyone was connecting remotely and remotely accessing multimodal digitized material (written, audio, visual) revealed the benefit of multimodality for all students. Because all exams had to be administered remotely, for all students, it was no longer only students who required accommodations for written exams to use personal computers for writing, it was all students. We hope that these are Universal Design features which Universities should retain after the Covidrelated emergency is over.

\section{References}

[1] Bencini GML, Garofolo I, Arenghi A. Implementing Universal Design and the ICF in Higher Education: Towards a Model That Achieves Quality Higher Education for All. In Craddock G, Doran C, McNutt L, Rice D, editors. Proceedings of Universal Design and Higher Education in Transformation Congress (UDHEIT2018): learning from the past, designing for the future. Studies in Health Technologies and Informatics, 2018 Oct 30 - Nov 2. Amsterdam, Berlin, Washington DC: IOS Press; 2018. pp.464-472.

[2] WHO (World Health Organization). International Classification of Functioning, Disability and Health (ICF-DH). Geneva; 2001.

[3] UN (United Nations). Convention on the Rights of Persons with Disabilities (A/R/61/106). New York; 2006.

[4] Arenghi A., Bencini G., Pavone M., Savarese, DaD in Università durante il lockdown: criticità e potenzialità (Distance learning in university during lockdown: critical aspects and possibilities). L'integrazione scolastica e sociale 19(3), Erickson; 2020, pp. 48-67. 